\title{
Pathophysiology of Stress
}

\author{
Nichole Rosenhagen, DVM \\ PAWS WildLIFE CENTER \\ SEATtLe, Washington
}

\begin{abstract}
Stress is an unavoidable reality for wild animals in captivity. In small bouts, its effects are minimal and can even be beneficial to an animal. However, long term stress is unequivocally detrimental to all species and can affect nearly every part of the body. Recognizing sources of stress and taking steps to mitigate its presence in wildlife rehabilitation facilities can hasten an animal's recovery and may even improve its overall survivability after release.
\end{abstract}

Keywords: Stress, glucocorticoids, cortisol, corticosterone

\section{STRESS}

The health implications of chronic stress have long been recognized in human medicine. Recently, a significant increase in the amount of research investigating how chronic stress affects animals has occurred. In spite of the negative connotation associated with the concept of stress, it is important to understand that stress is a natural part of life, and in small quantities, for short periods of time, the effects of stress on an individual may be beneficial and even convey a survival advantage. Therefore, the goal of stress management should be to minimize its presence rather than abolish it.

In order to fully evaluate stress, one must first comprehend what constitutes stress. Stress can be generally understood as the physiologic effects that occur in response to demanding stimuli. These stimuli, aptly named stressors, can be physical or psychological in nature. In the animal kingdom, everyday challenges like competition for food, defense of territory, and evasion of predators are natural stressors. However, despite the omnipresence of stressors, an individual's response to stress is variable and affected by a myriad of factors, including the animal's natural history, immediate environment, social hierarchy, and genetics. Thus, an event that triggers a stress response in one animal may go unnoticed in another.

Nicki Rosenhagen, DVM graduated from the University of Illinois College of Veterinary Medicine in 2015. After graduation, she worked for two years in the University's Wildlife Medical Clinic. She is now a full-time veterinarian at the PAWS Wildlife Center in Lynnwood, WA.

\section{GLUCOCORTICOIDS}

Glucocorticoids are some of the main hormones implicated in an animal's stress response. Yet these hormones, like stress itself, are a natural part of daily life for all animals, and their circulating levels fluctuate with the animal's circadian rhythm. This cycle varies by species and individual, and can be impacted by sleep pattern, light exposure, feeding times, and, of course, exposure to stressors (Polonski et al 2016).

Once a stressor is recognized, the brain sends a signal to activate the hypothalamic-pituitary-adrenal (HPA) axis, which leads to the release of glucocorticoids from the cortex of the adrenal gland (Polonski et al 2016). The primary glucocorticoid produced (cortisol or corticosterone) depends on the type of animal; most mammals release cortisol while birds, reptiles and amphibians release corticosterone. (Reeder and Kramer 2005).

In moderation, the effects of these hormones lead to rapid changes in the body that are often crucial to survival. Glucocorticoids increase blood glucose levels to provide the individual with an immediate source of energy. They also increase cardiac output, increase blood pressure, and dilate the bronchioles, the combined effect of which works to enhance oxygen and nutrient delivery to tissues that may be involved in an impending 'fight or flight' response. Finally, there is evidence that glucocorticoids enhance awareness, improve reflex time and even work with other hormones to create 'flash bulb' memories as a means to remember, and hopefully avoid, a particular stimulus in the future (Polonski et al 2016). The impact of glucocorticoids on the body's physiology is extensive; thus, the above review is by no means exhaustive but rather a brief overview of some of the most important changes.

While the acute effects of glucocorticoids are favorable, in excess these same hormones can alter the body's physiology in severe and sometimes even life-threatening ways. 


\section{PATHOPHYSIOLOGY OF CHRONIC STRESS}

As with normal physiology, there is no one size fits all pathophysiology for every animal. However, there is a vast amount of research to prove that chronic stress in any species is associated with increased morbidity and mortality. The following paragraphs highlight some of the most common consequences of chronic stress.

Glucocorticoids are so named because of their role in glucose regulation. With a stress-induced increased energy demand, the body shifts to a catabolic state, wherein glucocorticoids produce readily available glucose via tissue breakdown. For short, intermittent periods, catabolism is beneficial, but long-term catabolism leads to extensive physical impairment. The catabolic effects of glucocorticoids decrease production of growth hormone, inhibit DNA and protein synthesis, stimulate lipolysis, and trigger bone reabsorption (Polonski et al 2016). Consequently, wound healing is delayed, vital fat stores are depleted, and bones become weak and brittle. The results are severe in any animal, but can be especially pronounced in young, growing animals.

One of the most well-known repercussions of chronic stress is suppression of the immune system. Glucocorticoids naturally reroute or decrease production of some types of white blood cells while simultaneously decreasing the lifespan and efficacy of others (Marketon et al 2008). Scientists have even proclaimed that the immunological changes observed during chronic glucocorticoid exposure are similarly observed during natural aging processes (Macallan 2006). There are two main hypotheses as to why this may be beneficial to the animal in the short-term. The first is that the body may be reallocating the resources that would normally support the immune system to regions of the body that support immediate physical performance, thereby improving the animal's ability to flee or defend itself. The second hypothesis is that downregulating the immune system may decrease the chance of autoimmune damage. In the event of cell and tissue damage, self-antigen is released, which stimulates inflammation. During periods of intense physical stress and ensuing cellular trauma, excessive amounts of free self-antigen can hyperactivate the immune system and prime it to target healthy tissue (Martin 2009). Generally, the first hypothesis is more supported and accepted, though both ideas hold merit. Regardless of the reason, chronic alteration of immune function increases the likelihood of the animal contracting secondary infections from microorganisms that may otherwise be harmless in a healthy animal. Research has also revealed that animals in stressful conditions may be more likely to shed infectious organisms, and that some bacteria increase their virulence in response to stress hormones (Jones et al 2001). Therefore, the opportunity for secondary infections is facilitated not only by the animal's weakened immune system but also by the presence of larger numbers of more dangerous organisms.

As part of their role in redirecting the immune cells, glucocorticoids also inhibit the production of prostaglandins. Prostaglandins are lipid compounds that play many integral roles in a healthy animal. When their production is blocked from sustained glucocorticoid exposure, adverse changes occur in the body. Clinically, one of the most notable effects results from increased gastric acid and decreased gastric mucus; this can lead to gastrointestinal upset, ulcerations, and altered nutrient assimilation (Polonski et al 2016). In extreme cases, ulcers have been noted to develop within hours of exposure to a stressor (Wiklund et al 1996; Suleman et al 2004).

Chronic stress can be detrimental to an animal's offspring as well as the individual experiencing it. Glucocorticoids have been shown to reduce clutch and litter sizes, and can decrease the size and overall body condition of juveniles. In affected females, glucocorticoids can even inhibit ovulation altogether (Braastad 1998; Dobson et al 2000).

In the veterinary field, practitioners are often focused on the physical effects of disease, but chronic stress can also cause damaging psychological changes that should not be overlooked. High levels of circulating glucocorticoids affect an animal's memory and its ability to learn; they can also induce feelings of panic and fatigue that can manifest as aggression and selfinjurious behavior (Polonski et al 2016). These animals may lose adaptive coping strategies and the ability to appropriately respond to stressful stimuli, potentially impacting their success after release back to the wild.

There are many other, less commonly recognized effects of chronic stress. Corneal ulcerations and cataracts, cardiac arrhythmias, and anemia may be attributed to excessive glucocorticoid exposure (Polonski et al 2016). Likewise, chronically stressed animals have been shown to have reduced antioxidant capacity, altered cell longevity, and enhanced neoplastic cell growth (Marketon et al 2008). To further illuminate the effects of stress on cancer cells, mice with prostatic neoplasia were divided into groups and exposed to different levels of stress. All mice were treated with the same chemotherapeutic drug. In the relaxed control mice, the drug destroyed the cancer cells and inhibited tumor growth. Conversely, in mice that were exposed to repeated stressors, not only was the chemotherapy 
ineffective but the tumors became larger. In a subsequent model, mice with prostate cancer were all exposed to stressful stimuli that lead to tumor growth. Some of those same animals then received drugs to block the effects of glucocorticoids in the body, and tumor growth ceased (Hassan et al 2013). Cancer is uncommon in free-living wild animals, but this research is a startling revelation of the vast potential effects of unregulated stress levels.

\section{IMPLICATIONS FOR WILDLIFE REHABILITATION}

Many of the stressors for wild animals in captivity are unavoidable. Pain from physical injuries, contact with humans, and an unnatural environment are all sources of inherent stress in a hospital. Other stressors may not be as obvious. Altered light cycles, proximity to predators, and lack of stimulation can also be sources of stress. Some of these can be easily removed from the animal's environment; others can at least be mitigated. Highlighted here are some suggestions for alleviating the stress associated with captivity.

- Minimize frequency and duration of handling

- Provide visual barriers

- Keep the environment quiet

- Provide appropriate housing for the species, including areas to retreat and hide

- House prey animals away from predators

- Offer natural sources of food

- Combine healthy social animals or provide companionship in the form of mirrors, stuffed animals, etc.

- Provide enrichment

- Provide analgesics if the animal is painful

The reader should remember that wild animals may not always exhibit obvious signs of stress. A calm or stoic demeanor in an animal should not be taken as evidence of a relaxed state. Handling, talking to and looking at patients can elicit a physiologic stress response, even if there are no outward signs of distress. Wild animals also naturally mask pain, which can make its identification challenging for the rehabilitator. However, if the condition would warrant pain management in a cat or dog, it is safe to assume the wild animal would also benefit from some form of analgesia.

The dangerous effects of chronic stress on wildlife in rehabilitation settings are extensive. Excessive stress slows wound healing, increases the chance of secondary infections, and generally leads to a poorer quality of life for the animal. This is turn may manifest as prolonged recovery and/or decreased survivability after release. In extreme cases, stress can lead to death.
Therefore, wildlife professionals are encouraged to identify potential sources of stress in rehabilitation facilities and explore methods to reduce its presence, for the sake of the animals and their caretakers.

\section{LITERATURE CITED}

Braastad, B. O. 1998. Effects of Prenatal Stress on Behaviour of Offspring of Laboratory and Farmed Mammals. Applied Animal Behaviour Science. 61(2): 159-80.

Dobson, H., and R. F. Smith. 2000. What is Stress, and How Does it Affect Reproduction? Animal Reproduction Science 60-61: 743-752.

Hassan, S., Y. Karpova, D. Baiz, D. Yancey, A. Pullikuth, A. Flores, T. Register, J. M. Cline, R. D'Agostino, N. Danial, S. R. Datta, and G. Kulik. 2013. Behavioral Stress Accelerates Prostate Cancer Development in Mice. Journal of Clinical Investigation. 123(2): 874-886

Jones, P. H., J. M. Roe, and B. G. Miller. 2001. Effects of Stressors on Immune Parameters and on the Faecal Shedding of Enterotoxigenic Escherichia Coli in Piglets Following Experimental Inoculation. Research in Veterinary Science. 70(1): 9-17.

Macallan, B. M. 2006. Dasyurid Marsupials as Models for the Physiology of Ageing in Humans. Australian Journal of Zoology. 54(3): 159.

Marketon, J., I. Webster, and R. Glaser. 2008. Stress Hormones and Immune Function. Cellular Immunology. 252(1-2): 16-26.

Martin, L. B. 2009. Stress and Immunity in Wild Vertebrates: Timing Is Everything. General and Comparative Endocrinology. 163(1-2): 70-76.

Polonski, K. S., P. R. Larson, and H. M. Kronenberg. 2016. The Adrenal Cortex. Pp. 489-555 in Williams Textbook of Endocrinology. By Shlomo Melmed. 13th ed. Elsevier: Philadelphia, PA.

Reeder, D. M., and K. M. Kramer. 2005. Stress in Free-Ranging Mammals: Integrating Physiology, Ecology, and Natural History. Journal of Mammalogy. 86(2): 225-235.

Suleman, M. A., E. Wango, R. M. Sapolsky, H. Odongo, and J. Hau. 2004. Physiologic Manifestations of Stress from Capture and Restraint of Free-Ranging Male African Green Monkeys (Cercopithecus Aethiops). Journal of Zoo and Wildlife Medicine. 35(1): 20-24.

Wiklund, E., G. Malmfors, K. Lundstrom, and C. Rehbinder. 1996. Pre-slaughter Handling of Reindeer Bulls (Rangifer tarandus tarandus L.): Effects on Technical and Sensory Meat Quality, Blood Metabolites and Muscular and Abomasal Lesions. Rangifer. 16: 109-117. 